\title{
Sensitivity of female secondary characters in the crustacean isopod, Armadillidium vulgare, to the androgenic gland hormone: a hypothesis on the existence of an ovary-differentiating factor
}

\section{Sachiko Suzuki}

\begin{abstract}
The period during postembryonic development in which the androgenic gland hormone can masculinize female secondary characters was studied, with emphasis on the sexually undifferentiated period, in Armadillidium vulgare. Genetic females were implanted with androgenic glands at various developmental stages to induce masculinization. Instead of injection of androgenic gland extracts, androgenic glands were implanted after treatment with ethanol.
\end{abstract} Each genetic female received a total of three gland implants at stages 2-8. Masculinization of female endopodites of the first pair of abdominal legs was used to show sensitivity to the androgenic gland hormone. All the female recipients showed at least some masculinization of their endopodites at the first and second molts after receiving the implants regardless of the developmental stages. However, masculinization of their endopodites did not continue at subsequent molts. Only the genetic females, which received implants during the sex differentiation period, had endopodites that had fully developed into male copulatory organs. These results show that the secondary sexual characters of genetic females are sensitive to the androgenic gland hormone not only during the sex differentiation period but also during the sexually undifferentiated period. The effects of the androgenic gland implants on female secondary characters and gonadal development are discussed and an ovary-differentiating factor is hypothesized.

\section{Introduction}

In male crustaceans, the androgenic gland hormone (AGH) has been considered to be responsible for differentiation of all the male sexual characters (reviews by Charniaux-Cotton, 1960; CharniauxCotton \& Payen, 1985; Katakura, 1984, 1989; Payen, 1991; Charniaux-Cotton et al., 1992; Hasegawa et al., 1993; Sagi et al., 1997). In Armadillidium vulgare, gonadal sex differentiation can be altered by implantation of androgenic glands (AGs) in females (Katakura, 1959,1961; Hasegawa \& Katakura, 1985; Suzuki \& Yamasaki, 1997) and by andrectomy (removal of AGs) with partial gonadectomy of males (Suzuki \& Yamasaki, 1991). In this species, gonadal sex can also be transformed from female to male by implants of ethanol-treated androgenic gland ( $t-A G$ ) during the sex differentiation period (Suzuki \& Yamasaki, 1998; Suzuki, 1999). However, when genetic females receive $t$-AG implants during the sexually undifferentiated period, gonadal sex is not altered (Suzuki, 1999). The effects of t-AG implants on female secondary characters during the sex differentiation period have been reported (Suzuki \& Yamasaki, 1998), but they have not yet determined for the sexually undifferentiated period.

The aim of this study was to determine whether the secondary sex characters of 
genetic female A.vulgare can be masculinized by AGH at stages 2-3 during the sexually undifferentiated period. Genetic females received the t-AGs implants at stages $2-8$ of postembryonic development instead of giving an AGH injection. The effect of t-AG implants on gonadal development has already been reported (Suzuki, 1999). Herein the effect on female secondary characters is presented. In this study, masculinization of female endopodites was used as an indicator of sensitivity of female secondary characters to AGH.

\section{Materials and Methods}

\section{Animals}

All the genetic females and genetic males of $A$. vulgare used in this study were raised in the laboratory (Suzuki, 1999). Genetic females (ZW) were used at stages 2-8 as recipients for t-AG implantations in the experimental and control groups (Suzuki, 1999). Genetic males (ZZ) were also used at stage 4 as a control group (Suzuki, 1999). Each stage was defined with the number of molts (Suzuki \& Yamasaki, 1995). The specimens of stage 2 underwent the first ecdysis after having been released from the marsupium. The relationship between gonadal development and the stage of postembryonic development has previously been described for A. vulgare (Suzuki \& Yamasaki, 1995).

\section{Ethanol-Treated AG Implantation}

Fresh AGs were treated twice with $80 \%$ ethanol for $3 \mathrm{~min}$ each time, as described by Suzuki (1999). These t-AGs were immediately implanted into recipients through a hole made in the tergite of the fourth or sixth thoracic segment under a dissecting microscope (Suzuki, 1999). Experimental specimens of each group were given an implant of one t-AG, three times at 3 or 4 day intervals, at developmental stages $2(\mathrm{~N}=21$ specimens), 3 (22), 4 (47), 5 (29), 6 (25), 7 (21) or 8 (16). Each experimental female received a total of three t-AG implants, as shown previously (Suzuki, 1999). As a control, genetic females $(\mathrm{N}=12)$, received implants of ethanol-treated muscle instead of t-AG at stage 4. A second control group, genetic males $(\mathrm{N}=15)$, received implants of $\mathrm{t}$-AG at stage 4 .

\section{Observation of Secondary Sex Characters}

After the implantations, all of the specimens were reared until 120 days of postembryonic development had passed (Suzuki, 1999). At the termination of the experiment, the specimens were sacrificed to observe the masculinized levels of the secondary sex characters. The length of each endopodite was measured with the aid of an ocular micrometer mounted in a compound microscope, and the morphology was compared (Suzuki \& Yamasaki, 1998) with that of the control females and males.

\section{Statistical analysis}

The differences of endopodite lengths were evaluated for statistical significance with Student's t-test.

\section{Results}

During the experimental period the genetic females molted successfully, as shown previously (Suzuki, 1999). The control females, which received the implants of muscle at stage 4 , did not masculinize their endopodites (Figs. 1A, 2A). The control males, which received the $t$ AG implants at stage 4, elongated their endopodites at each molt and formed the copulatory organs with penes (Figs. 1B, $3 \mathrm{~A})$, indicating that the $\mathrm{t}-\mathrm{AG}$ implants did not prevent normal development of male copulatory organs.

All of the genetic female recipients showed masculinized endopodites after receiving the t-AG implants. The masculinized endopodites could be divided into two types: an incompletely masculinized type (Figs. 1A, 2) and a completely masculinized type (Figs. 1B, 3). The former type 
Incompletely masculinized type

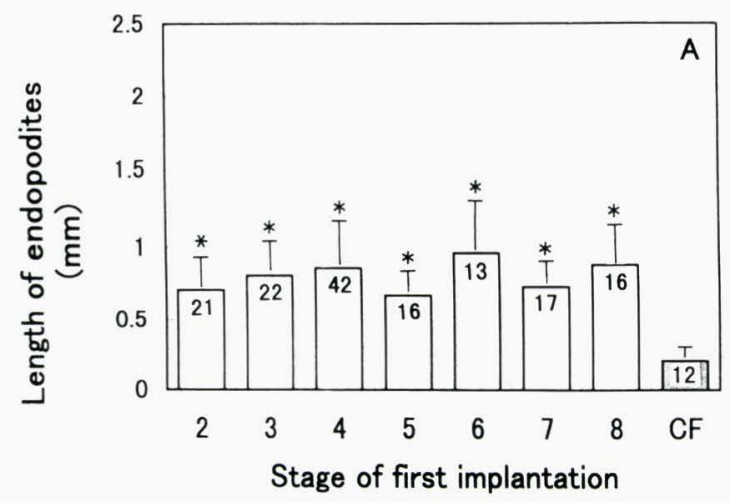

Completely masculinized type

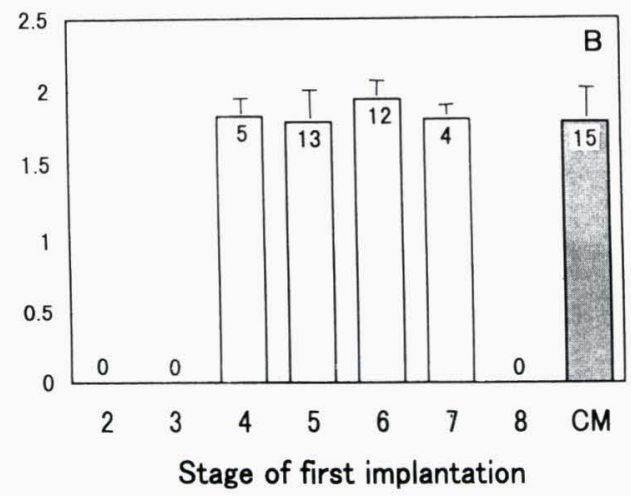

Fig. 1. A comparison of masculinized endopodites induced by implants of ethanol-treated androgenic glands (t-AGs). Experimental females received t-AG implants at developmental stages 2-8. Control females (CF) received implants of ethanol-treated muscle at stage 4. Control males (CM) were implanted with t-AGs at stage 4 . At 120 days of postembryonic development, the lengths of the endopodites in all the specimens were measured. Vertical bars represent the mean \pm SD of endopodite lengths. The number in each column is the number of animals measured. 0 indicates no observation. *, $P<0.001$ compared with control females.

showed incomplete transformation of the female endopodites with small penes. The latter type showed full transformation of the female endopodites into male copulatory organs. When genetic females received t-AG implants at stages $2-3$ during the sexually undifferentiated period, their endopodites became elongated at the first and second post-implanted molts. However, their development stopped at subsequent molts, at incompletely masculinized levels with small penes (Figs.1A, 2B). That is, these females of stages $2(\mathrm{~N}=21 / 21,100 \%)$ and $3(22 /$ $22,100 \%)$ at which the t-AG implants were given had endopodites that were only incompletely masculinized by the implants. On the other hand, when genetic females received implants at stages 4-7 during the sex differentiation period, both incomplete and complete types were observed. The recipient females of stages 4 $(\mathrm{N}=42 / 47,89.4 \%), 5(16 / 29,55.2 \%), 6(13 /$ $25,52 \%)$ and $7(17 / 21,81 \%)$ were induced to elongate their endopodites at the first and second post-implanted molts but masculinization stopped at subsequent molts (Figs. 1A, 2C). Therefore, they had incompletely masculinized endopodites. The remaining recipient females were induced to elongate their endopodites continuously at subsequent molts when the tAG implants were given at stages $4(\mathrm{~N}=$ $5 / 47,10.6 \%), 5(\mathrm{~N}=13 / 29,44.8 \%), 6(\mathrm{~N}=$ $12 / 25,48 \%)$ and $7(4 / 21,19 \%)$ (Figs. $1 \mathrm{~B}$, $3 \mathrm{~B}, 3 \mathrm{C})$. That is, they had completely masculinized endopodites. When genetic females received t-AG implants at stage 8 , all ( $\mathrm{N}=16 / 16,100 \%)$ had incompletely masculinized endopodites (Fig.1A), no completely masculinized ones were observed (Fig.1B).

The lengths of the masculinized endopodites of the experimental groups were compared with those of the control groups of both sexes. Even the incompletely masculinized endopodites were significantly longer $(P<0.001)$ than those of the control females (Fig. 1A), showing that the female endopodites could be masculinized by the t-AG implants which were given not only during the sexual differentiation period but also during the sexually undifferentiated period. The completely masculinized endopodites in the experimental groups, given t-AG im- 

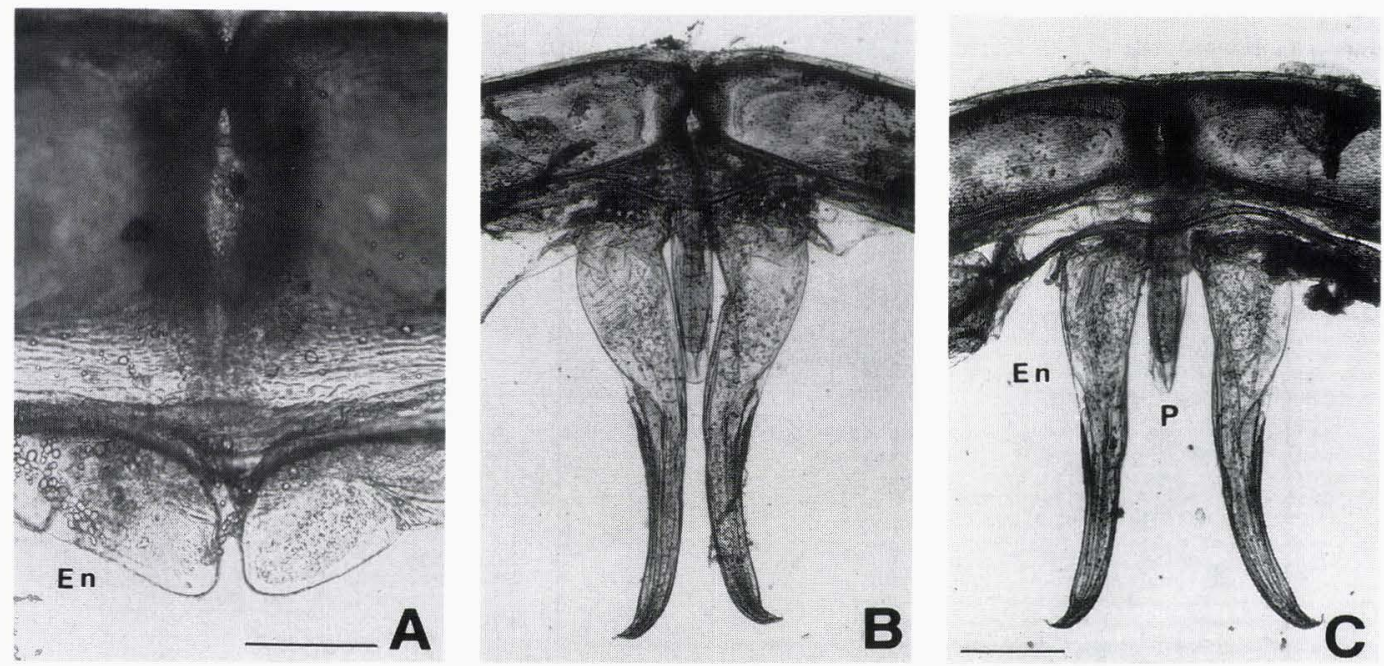

Fig. 2. Micrographs showing incompletely masculinized endopodites. A, control female given muscle implants at stage 4 . Experimental female that received the first t-AG implant at (B) stage 3 or (C) stage 5. En,endopodite; P, penis. Scale bars represent $200 \mu \mathrm{m}$.
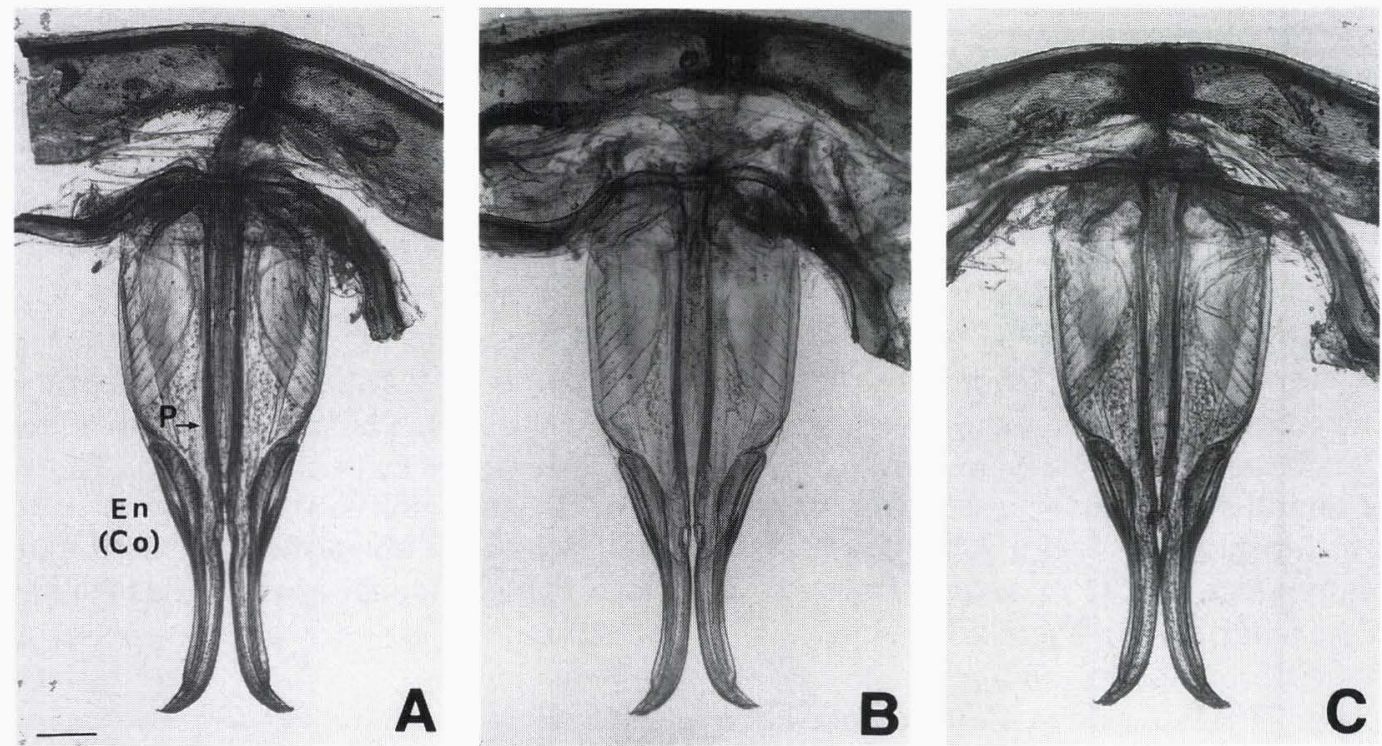

Fig. 3. Micrographs showing completely masculinized endopodites. A, control male given the tAG implants at stage 4. Experimental female received the first implant of a t-AG at (B) stage 4 or (C) stage 6. En, endopodite (male copulatory organs); P, penis. Scale bar represents $200 \mu \mathrm{m}$.

plants at stages $4-7$, showed no significant difference when compared with the control male group (Fig. 1B), exhibiting complete transformation into male copulatory organs.
Discussion

In normal postembryonic development, the endopodites of the first pair of abdominal legs of males begin to develop at stage 5. Male endopodites continuously 


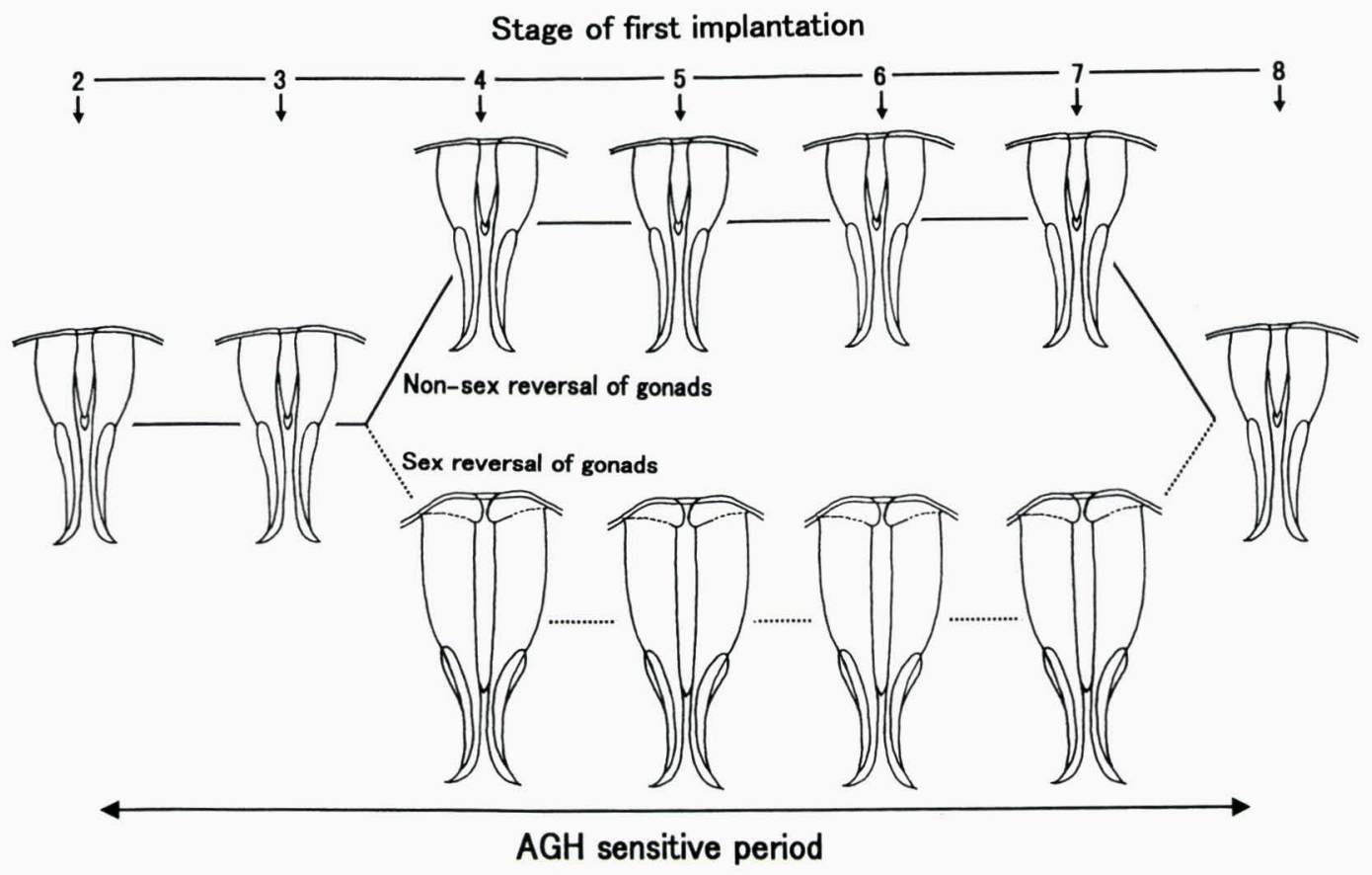

Fig. 4. The effects of t-AG implants on the development of female endopodites. The results are summarized here. Small arrows show the developmental stage at which genetic females received the first t-AG implant. Each drawing presents the typical morphology of masculinized endopodites induced by t-AG implants. Gonadal development shown in this figure was reported previously (Suzuki, 1999).

elongate at each molt until stage 8 and form the male copulatory organs (Suzuki \& Yamasaki, 1995). In normal females the endopodites do not show such elongation through postembryonic development. If females are sex reversed after stage 5 by implants of fresh AGs (Suzuki \& Yamasaki, 1997) or t-AGs (Suzuki \& Yamasaki, 1998), their endopodites will have developed into male copulatory organs. These previous experiments have suggested that female endopodites are sensitive to AGH during the sex differentiation period in this species.

In the present study, masculinization of endopodites was induced in all experimental females at the first and second molts after receiving t-AG implants at developmental stages 2-8. At subsequent molts no further elongation of their endopodites occurred in many recipients (Fig. 1A). As the result, they had incom- pletely masculinized endopodites. At the end of the experiment, the gonadal sex of the recipient females was determined (Suzuki, 1999). These females were not sex reversed by the t-AG implants. Therefore, the t-AG implants into genetic females induced incomplete masculinization of the endopodites regardless of the implanted stage of postembryonic development (Fig. 4).

On the other hand, some of the recipient females that received the $\mathrm{t}-\mathrm{AG}$ implants at stages $4-7$ were induced to masculinize their endopodites completely (Figs. 1B). As shown in the previous study, these females had transformed their ovaries into testes (Suzuki, 1999). As a result of sex reversal of gonads, they could elongate their masculinized endopodites continuously at each molt because of AGH secreted from the newly developed AGs (Suzuki \& Yamasaki, 1998). 


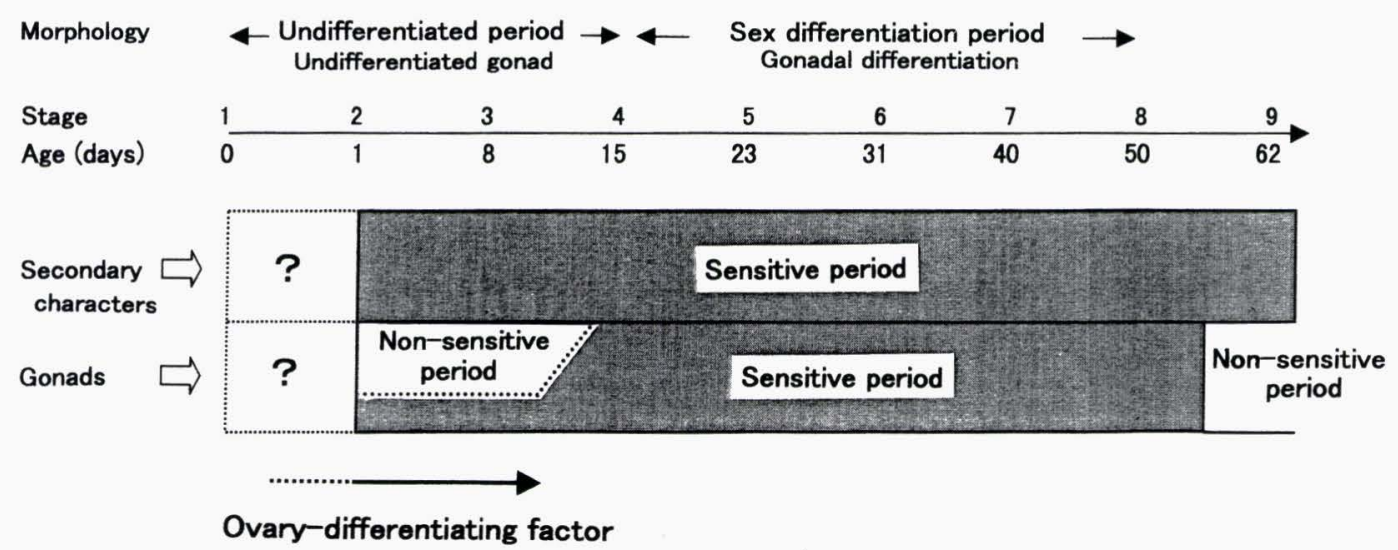

Fig. 5. Changes of sensitivity to AGH through postembryonic development in female A. vulgare. Gonadal differentiation (Suzuki \& Yamasaki, 1995) and age (days) of postembryonic development (Suzuki, 1999) have been shown for this species. The sensitive period of the secondary sex characters was determined in this study (Figs. 1, 4). The sensitive period of the female gonads was reported previously (Suzuki, 1999). An ovary-differentiating factor is hypothesized to exist in the anterior regions of the undifferentiatesd gonads of genetic females of A. vulgare.

Furthermore, they could transform their endopodites into male copulatory organs, the completely masculinized type (Fig. 4).

In this study two types (Figs. 1A, 1B) of masculinized endopodites were observed in the recipient females, depending on whether the gonad had undergone sex reversal (Fig. 4). The degree of masculinization of the female endopodites does not depend on the developmental stages at which the t-AGs are implanted. This fact shows that genetic females are sensitive to the masculinizing effect of AGH on their secondary sex characters not only during the sex differentiation period but also during the sexually undifferentiated period (Fig. 5).

Our previous observations on gonadal development have shown that female gonads were sex reversed by the t-AG implants during stages 4-7 (Suzuki \& Yamasaki, 1998; Suzuki, 1999), suggesting that female gonads are sensitive to AGH during the sex differentiation period. In contrast, undifferentiated gonads of genetic females were not sex reversed by t-AG implants during the sexually undifferentiated period (Suzuki, 1999). However, the posterior ends of the undif- ferentiated gonads were masculinized by t-AGs, having a rudimentary vas deferens (Suzuki, 1999). Based on our previous observations, the anterior regions of the undifferentiated gonads are probably not sensitive to AGH but the posterior regions are sensitive, as shown in Figure 5.

It is now generally accepted in vertebrates that the undifferentiated gonads of both sexes are sexual bipotent and can develop as either a testis or ovary (Jost, 1970; Nagai, 1992; Clinton, 1998). In the crustacean isopod A. vulgare, Katakura (1989) has reported that the primordial gonads of both sexes are highly sensitive to AGH in the early stage of postembryonic development. Figure 5 shows the sensitive periods for AGH in this species. The organs that form the endopodites (secondary sex characters) in genetic females are sensitive to AGH during not only the undifferentiated period but also during the sex differentiation period. This fact suggests that female secondary characters possess receptors for $\mathrm{AGH}$ throughout postembryonic development. On the other hand, female gonads are highly sensitive to AGH and sexual bipotent during the sex differentiation pe- 
riod (Suzuki, 1999). However, during the sexually undifferentiated period, the anterior regions of undifferentiated gonads are not sensitive, but the posterir regions are sensitive, as mentioned above (Fig. 5).

In this species, primordial testes are located on the anterior regions of the gonads in both sexes (Suzuki \& Yamasaki, 1995 ) and from these regions, sex-reversed genetic females develop their testes during the sex differentiation period (Suzuki \& Yamasaki, 1997). Whether the anterior regions of undifferentiated gonads are not sensitive to AGH or just lack AGH receptors remains to be determined, and leaves open the possibility that an ovary-differentiating factor exists in the anterior regions of female gonads during the sexually undifferentiated period (Fig. $5)$. This factor may be antagonistic to $\mathrm{AGH}$ receptors and may be blocking testis development even if exogenous AGH is injected into genetic females. Because of this feminizing factor, undifferentiated gonads would develop into an ovary without morphological sex differentiation. Once the direction of gonadal differentiation is toward an ovary at the end of stage 3 , this factor will no longer be produced. At later stages the gonads of females are sensitive to $\mathrm{AGH}$, and then can be induced to develop primordial testes (Fig. 5). Therefore, AGH is capable of transforming female gonads into functional male gonads during the sex differentiation period. Female gonads may progressively lose their sensitivity to AGH as the developmental stage proceeds. Females of stage 9 are incapable of developing testes from their gonads (Katakura, 1989; Suzuki \& Yamasaki, 1997), suggesting that the AGH receptors in female gonads have a critical period for testis development, gonadal sex reversal no longer being possible at and after stage 9 of postembryonic development.

It has reported that this species usually has a ZW female-ZZ male sex determining system (Hasegawa \& Katakura,
1983; Legrand et al., 1987; Katakura, 1989; Juchault \& Rigaud, 1995; Rigaud et al., 1997). Furthermore, a female determining gene, which is located on the $\mathrm{W}$ chromosome, has been hypothesized to inhibit differentiation of the AG (Katakura, 1989; Rigaud et al., 1997). The hypothesized ovary-differentiating factor may be one of the feminizing factors which are produced by female sex-differentiating genes. The AGH of this species has recently been identified and purified (Okuno et al., 1997, 1999; Martin et al., $1998,1999)$. To clarify the regulatory mechanism of sex differentiation, the exact role of the male sex hormone, AGH, should also be elucidated with respect to gonadal sex differentiation in male $A$. vulgare.

\section{Acknowledgments}

The author expresses her gratitude to Professor Milton Fingerman (Tulane University) for his many helpful suggestions during the course of this investigation. The author also expresses her gratitude to Professor Gilbert Martin (Poitiers University) for many valuable comments on the manuscript.

\section{Literature Cited}

Charniaux-Cotton, H., 1960. Sex determination. In: T. H. Waterman, (ed.), The Physiology of Crustacea, Volume 1, pp. 411-447. Academic Press, New York.

, \& Payen, G., 1985. Sexual differentiation. In: D. E. Bliss and L.H. Mantel,(eds.), The Biology of Crustacea, Volume 9, pp. 217-299. Academic Press,New York.

, Payen, G. G., \& Ginsburger-Vogel, T., 1992. Arthropoda-Crustacea: Sexual Differentiation. In: K.G. Adiyodi \& R.G. Adiyodi, (eds.), Reproductive Biology of Invertebrates, Volume 5, pp. 281-323. Wiley, Chichester/ New York.

Clinton, M., 1998. Sex determination and gonadal development: a bird's eye view. Journal of Experimental Zoology, 281: 457-465.

Hasegawa, Y., \& Katakura, Y., 1983. Masculinization of WW females in the Isopod Crustacean, Armadillidium vulgare. Annotationes Zoological Japonenses, 56: 
163-166.

, \& Katakura, Y., 1985. Masculinization of female by the newly-formed Androgenic glands in the ZW and WW females of the isopod crustacean, Armadillidium vulgare. Zoological Science, 2: 419-422.

— Hirose, E., \& Katakura, Y., 1993. Hormonal control of sexual differentiation and reproduction in Crustacea. American Zoologist, 33: 403-411.

Jost, A., 1970. Hormonal factors in the sex differentiation of the mammalian foetus. Philosophical Transactions of the Royal Society of London, Ser.B, 259: 119-130.

Juchault, P., \& Rigaud, T., 1995. Evidence for female heterogamety in two terrestrial crustaceans and the problem of sex chromosome evolution in isopods. Heredity, 75 : 466-471.

Katakura, Y., 1959. Masculinization through implanting testes into the female Armadillidium vulgare, an isopod crustacean. Proceedings of the Japan Academy, 35: 95-98.

- , 1961. Hormonal control of development of sexual characters in the isopod crustacean, Armadillidium vulgare. Annotationes Zoological Japonenses, 34: 60-71.

$\longrightarrow$, 1984. Sex differentiation and androgenic gland hormone in the terrestrial isopod Armadillidium vulgare. Symposium of Zoological Society of London, 53: 127-142.

- 1989. Endocrine and genetic control of sex differentiation in the malacostracan Crustacea. Invertebrate Reproduction and Development, 16: 177-182.

Legrand, J. J., Legrand-Hamelin, E., \& Juchault, P., 1987. Sex determination in crustacea. Biological Review, 62: 439-470.

Martin, G., Sorokine, O., Moniatte, M., \& Van Dorsselaer, A., 1998. The androgenic hormone of the crustacean isopod Armadillidium vulgare. Annals of the New York Academy of Sciences, 839: 111-117.

$\longrightarrow, \longrightarrow, 1$, Bulet, P., Hetru, C., \& Van Dorsselaer, A., 1999. The structure of a glycosylated protein hormone responsible for sex determination in the isopod, Armadillidium vulgare. Europian Journal of Biochemistry, 262: 727-736.

Nagai, Y., 1992. Primary sex determination in mammals. Zoological Science, 9: 475-498.

Okuno, A., Hasegawa, Y., \& Nagasawa, H., 1997. Purification and properties of androgenic gland hormone from the terrestrial isopod Armadillidium vulgare. Zoological
Science 14: 837-642.

— , Hasegawa, Y., Ohira, T., Katakura, Y., \& Nagasawa, H., 1999. Characterization and cDNA cloning of androgenic gland hormone of the terrestrial isopod Armadillidium vulgare. Biochemical Biophysical Research Communications, 249: 187-190.

Payen, G. G., 1991. Roles of androgenic gland hormone in determining the sexual characters in Crustacea. In: A. P. Gupta, (ed.), Morphogenetic hormones of Arthropods,Volume 3, pp. 431-452. Rutgers Univ. Press, New Brunswick, NJ.

Rigaud, T., Juchault, P., \& Mocquard, J.-P., 1997. The evolution of sex determination in isopod crustaceans. BioEssays, 19: 409416.

Sagi, A., Snir, E., \& Khalaila,I., 1997. Sexual differentiation in decapod crustaceans: Role of the androgenic gland. Invertebrate Reproduction and Development, 31: 55-61. Suzuki, S., 1999. Androgenic gland hormone is a sex-reversing factor but can not be a sexdetermining factor in the female crustacean isopods Armadillidium vulgare. General and Comparative Endocrinology, 115: 370-378.

— \& Yamasaki, K., 1991. Sex-reversal of male Armadillidium vulgare (Isopoda, Malacostraca, Crustacea) following andrectomy and partial gonadectomy. General and Comparative Endocrinology, 83: 375-378.

$\longrightarrow, \&-$, 1995. Morphological studies on sexual differentiation in Armadillidium vulgare (Isopoda: Armadillidae): Androgenic gland and male sexual characters. Crustacean Research, 24: 93-103.

$\longrightarrow, \& —, 1997$. Sexual bipotentiality of developing ovaries in the terrestrial isopod Armadillidium vulgare (Malacostraca, Crustacea). General and Comparative Endocrinology, 107: 136-146.

$\longrightarrow, \&-, 1998$. Sex reversal by implantations of ethanol-treated androgenic glands of female isopods, Armadillidium vulgare (Malacostraca, Crustacea). General and Comparative Endocrinology, 111: 367-375.

Address: Biological Laboratory, Kanagawa Prefectural College, Nakao 1-5-1, Asahiku, Yokohama 241-0815, Japan.

E-mail: suzuki.sachiko@nifty.com 